\title{
Effects of the spike timing-dependent plasticity on the synchronisation in a random Hodgkin-Huxley neuronal network
}

\author{
R. R. Borges ${ }^{1}$, F. S. Borges ${ }^{1}$, A. M. Batista ${ }^{1,2, *}$, E. L. Lameu ${ }^{1}$, R. L. \\ Viana $^{3}$, K. C. Iarosz ${ }^{4}$, I. L. Caldas ${ }^{4}$, M. A. F. Sanjuán ${ }^{5}$ \\ ${ }^{1}$ Pós-Graduação em Ciências, Universidade Estadual de Ponta Grossa, 84030-900, \\ Ponta Grossa, PR, Brazil. \\ ${ }^{2}$ Departamento de Matemática e Estatística, Universidade Estadual de Ponta Grossa, \\ 84030-900, Ponta Grossa, PR, Brazil. \\ ${ }^{3}$ Departamento de Física, Universidade Federal do Paraná, 81531-990, Curitiba, PR, \\ Brazil. \\ ${ }^{4}$ Instituto de Física, Universidade de São Paulo, 05315-970, São Paulo, SP, Brazil. \\ ${ }^{5}$ Departamento de Física, Universidad Rey Juan Carlos, Tulipán s/n, 28933 Móstoles, \\ Madrid, Spain.
}

\begin{abstract}
In this paper, we study the effects of spike timing-dependent plasticity on synchronisation in a network of Hodgkin-Huxley neurons. Neuron plasticity is a flexible property of a neuron and its network to change temporarily or permanently their biochemical, physiological, and morphological characteristics, in order to adapt to the environment. Regarding the plasticity, we consider Hebbian rules, specifically for spike timing-dependent plasticity (STDP), and with regard to network, we consider that the connections are randomly distributed. We analyse the synchronisation and desynchronisation according to an input level and probability of connections. Moreover, we verify that the transition for synchronisation depends on the neuronal network architecture, and the external perturbation level.
\end{abstract}

Keywords: plasticity, neuronal network, synchronisation

${ }^{*}$ Corresponding author: antoniomarcosbatista@gmail.com 


\section{Introduction}

The human brain contains about $10^{11}$ neurons [1], and each neuron is connected to approximately $10^{4}$ other neurons [2]. These connections called synapses, are arranged in a highly complex network. They are responsible for neuronal communication and can be classified into two categories: electrical and chemical synapses [3, 4]. In electrical synapses the transmission of information from one neuron to another is directly performed from the pre-synaptic cell to the post-synaptic cell via gap junctions. In chemical synapses, the process occurs via neurotransmitters, which cross the synaptic cleft and bind to receptors on the membrane of the synaptic cell [5]. Neurotransmitters may increase or decrease the probability of an action potential of a post-synaptic neuron, and the synapses are called excitatory or inhibitory, respectively [6]. Furthermore, the intensity of the chemical synapses can be modified, in other words, they can be minimised or potentiated [7]. The mechanism responsible for these adjustments is known as synaptic plasticity [8].

The synaptic plasticity, that is, the ability of synapses to weaken or strenthen over time [9] is an important property of the mammalian brain. In addition, the synaptic plasticity is also related to processes of learning and memory [10, 11]. This adjustment of the intensities of the chemical synapses can be correlated with phenomena of synchronisation of the neuronal firing [12].

The occurrence of synchronisation in some specific areas of the brain may be associated with some diseases, such as the epilepsy and the Parkinson's disease [13, 14, 15]. On the other hand, it is also responsible for some vital brain functions, such as processing of sensory information and motor function [16, 17].

Methods to suppress synchronisation have been proposed in neuroscience, as the introduction of external perturbations [18, 19, 20]. Tass and collaborators have verified the possibility of desynchronisation in hippocampal neuronal populations through coordinated reset stimulation [22]. Meanwhile, Popovych and collaborators have found that the introduction of a perturbation in a globally connected neuronal network combined with synaptic plasticity can provide a positive contribution to the firing synchronisation [20].

In this work, we study firing synchronisation in a random Hodgkin-Huxley neuronal network with plasticity according to spike timing-dependent plastic- 
ity (STDP). This synaptic plasticity model adjusts the connection strengths by means of the temporal interval between pre-synaptic and post-synaptic spikes [23, 24]. Bi and Poo have reported that the change in synaptic efficiency after several repetitions of the experiment is due to the time difference of firing [25, 26]. If one pre-synaptic spike precedes a post-synaptic spike, a long-term potentiation occurs, otherwise, a long-term depression appears [27].

A computational neuronal network specifies the connection architecture among neurons. A globally coupled Hodgkin-Huxley neuron model was also considered by Popovych and collaborators [20]. They studied the synchronisation behaviour considering STDP, and found that the mean synaptic coupling presents a dependence on the input level. In this work, we consider a random neuronal network with STDP, and input, where the connections are associated with chemical synapses [21]. One main result is to show that spike synchonisation in a neuronal network, depending on the probability of connections, can be improved due to spike timing-dependent plasticity. This improvement is also observed when an external perturbation is applied on the network. Another important result is that the orientation of the connections among neurons with a different spike frequency affect the synchronised behaviour.

This paper is organised as follows: in Section II we introduce the HodgkinHuxley neuronal model. In Section III, we show the random neuronal network. In Section IV, we study the synchronisation considering spike timingdependent plasticity. Finally, in the last Section, we draw the conclusions.

\section{Hodgkin-Huxley neuronal network}

\subsection{Hodgkin-Huxley neuronal model}

One of the most important models in computational neuroscience is the neuronal model proposed by Hodgkin and Huxley [28, 29]. In this model, the mechanism of generation of an action potential was elucidated in a series of experiments with the squid giant axon. They found three different ions currents consisting of sodium (Na), potassium (K) and leak (L) mainly due to chlorine. Moreover, there are voltage-dependent channels for sodium, potassium that control the entry and exit of these ions through the cell. The model is composed of a system of four coupled differential equations given by

$$
C \dot{V}=I-g_{\mathrm{K}} n^{4}\left(V-E_{\mathrm{K}}\right)-g_{\mathrm{Na}} m^{3} h\left(V-E_{\mathrm{Na}}\right)
$$




$$
\begin{aligned}
& -g_{\mathrm{L}}\left(V-E_{\mathrm{L}}\right), \\
\dot{n}= & \alpha_{n}(V)(1-n)-\beta_{n}(V) n, \\
\dot{m}= & \alpha_{m}(V)(1-m)-\beta_{m}(V) m, \\
\dot{h}= & \alpha_{h}(V)(1-h)-\beta_{h}(V) h,
\end{aligned}
$$

where $C$ is the membrane capacitance (measured in $\mu \mathrm{F} / \mathrm{cm}^{2}$ ), $V$ is the membrane potential (measured in $\mathrm{mV}$ ), the function $m(V)$ and $n(V)$ are the variable of activation for sodium and potassium, and $h(V)$ is the variable of inactivation for sodium. The functions $\alpha_{n}, \beta_{n}, \alpha_{m}, \beta_{m}, \alpha_{h}, \beta_{n}$ are given by

$$
\begin{aligned}
\alpha_{n}(V) & =0.01 \frac{10-V}{\exp \left(\frac{10-V}{10}\right)-1} \\
\beta_{n}(V) & =0.125 \exp \left(\frac{-V}{80}\right) \\
\alpha_{m}(V) & =0.1 \frac{25-V}{\exp \left(\frac{25-V}{10}\right)-1} \\
\beta_{m}(V) & =4 \exp \left(\frac{-V}{18}\right) \\
\alpha_{h}(V) & =0.07 \exp \left(\frac{-V}{20}\right) \\
\beta_{n}(V) & =\frac{1}{\exp \left(\frac{30-V}{10}\right)+1}
\end{aligned}
$$

The parameters $g$ and $E$ represent the conductance and reversal potentials for each ion. The constant $I$ is an external current density (measured in $\left.\mu \mathrm{A} / \mathrm{cm}^{2}\right)$ that determines a regime of a single spike $\left(I=0.0 \mu \mathrm{A} / \mathrm{cm}^{2}\right)$, or a scheme with periodic spikes $\left(I=9.0 \mu \mathrm{A} / \mathrm{cm}^{2}\right)$, as illustrated in Fig. 11(a) and (b), respectively. Moreover, the spikes frequency increases when the constant $I$ increases. For instance, $I=9.0 \mu \mathrm{A} / \mathrm{cm}^{2}$ and $I=10.0 \mu \mathrm{A} / \mathrm{cm}^{2}$ approximately correspond to $67 \mathrm{~Hz}$ and $70 \mathrm{~Hz}$, respectively. The parameters that we use in this work are presented in Table 1 [30].

\subsection{Network structure}

Computational models of neuronal networks depend on the architecture, which specifies how neurons are connected and how the dynamics is applied to each unit or node. In this work, we consider a random network, that is, the network is constructed by connecting neurons randomly [31, 32, 33]. 
Table 1: Parameters of the Hodgkin-Huxley neuronal model with a resting potential equal to $-65 \mathrm{mV}$.

\begin{tabular}{|c|c|c|}
\hline Description & Parameter & Values \\
\hline Membrane capacity & $C$ & $1 \mu \mathrm{F} / \mathrm{cm}^{2}$ \\
\hline Reversal potential for Na & $E_{\mathrm{Na}}$ & $120 \mathrm{mV}$ \\
\hline Reversal potential for $\mathrm{K}$ & $E_{\mathrm{K}}$ & $-12 \mathrm{mV}$ \\
\hline Reversal potential for $\mathrm{L}$ & $E_{\mathrm{L}}$ & $10.6 \mathrm{mV}$ \\
\hline Sodium conductance & $g_{\mathrm{Na}}$ & $120 \mathrm{mS} / \mathrm{cm}^{2}$ \\
\hline Potassium conductance & $g_{\mathrm{K}}$ & $36 \mathrm{mS} / \mathrm{cm}^{2}$ \\
\hline Leak conductance & $g_{\mathrm{L}}$ & $0.3 \mathrm{mS} / \mathrm{cm}^{2}$ \\
\hline External current & $I$ & $9.010 .0 \mu \mathrm{A} / \mathrm{cm}^{2}$ \\
\hline
\end{tabular}

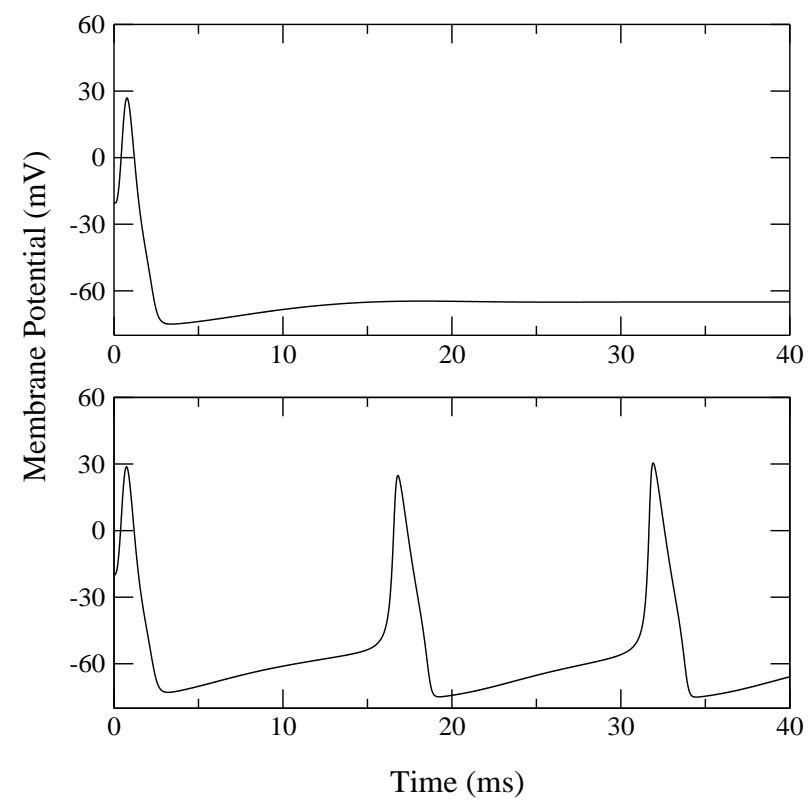

Figure 1: Dynamic firing in the Hodgkin-Huxley model, where we consider (a) $I=$ $0.0 \mu \mathrm{A} / \mathrm{cm}^{2}$ that shows a single spike with a subsequent resting state, and (b) $I=$ $9.0 \mu \mathrm{A} / \mathrm{cm}^{2}$ that presents a regime with periodic firing. 
Each connection is included with probability independent from every other connection. Figure 2 exhibits a schematic representation of the neuronal network considered in this work. Each neuron is connected to others by randomly chosen neurons with probability $p$. When $p=1$ we have a global network, where all neurons are connected.

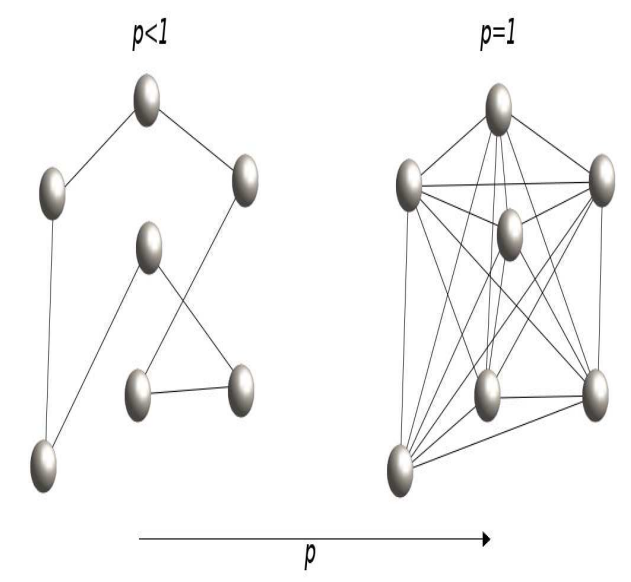

Figure 2: Schematic representation of a network in which each neuron is connected to others by randomly chosen neurons with probability $p$.

We consider a random neuronal network with chemical synapses where the connections are unidirectional, and the local dynamics is described by the Hodgkin-Huxley model. The network is given by

$$
\begin{aligned}
C \dot{V}_{\mathrm{i}}= & I_{i}-g_{\mathrm{K}} n^{4}\left(V_{\mathrm{i}}-E_{\mathrm{K}}\right)-g_{\mathrm{Na}} m^{3} h\left(V_{\mathrm{i}}-E_{\mathrm{Na}}\right)- \\
& g_{L}\left(V_{i}-E_{L}\right)+\frac{\left(V_{r}-V_{i}\right)}{\omega} \sum_{j=1}^{N} \varepsilon_{i j} s_{j}+\Gamma_{i},
\end{aligned}
$$

where $V_{i}$ is the membrane potential of neuron $i(i=1, \ldots, N), I_{i}$ is a constant current density randomly distributed in the interval [9.0,10.0], $\omega$ is the average degree connectivity, and $\varepsilon_{i j}$ is the coupling strength from the presynaptic neuron $j$ to the post-synaptic neuron $i$, that is, normally distributed with mean and variance equal to 0.1 and 0.02 , respectively [27]. We consider an external perturbation $\Gamma_{i}$, so that each neuron receives an input with a constant intensity $\gamma$ during $1 \mathrm{~ms}$. This input is applied with an average time interval around $14 \mathrm{~ms}$. This value is approximately the inter-spike interval of 
a single neuron. The neurons are excitatory coupled with a reversal potential $V_{r}=20 \mathrm{mV}[20]$. The post-synaptic potential $s_{i}$ is given by [34, 35]

$$
\frac{d s_{i}}{d t}=\frac{5\left(1-s_{i}\right)}{1+\exp \left(-\frac{V_{i}+3}{8}\right)}-s_{i} .
$$

\subsection{Spiking neurons synchronisation}

When identical neurons are coupled, the network may exhibit a complete synchronisation among spiking neurons, i.e., all neurons have identical time evolution of their action potential. We do not consider identical neurons here, and due to this fact, a complete synchronisation is not possible. However, a weak synchronisation may be observed.

As diagnostic of spikes synchronisation we use the order parameter given by [36, 37]

$$
R=\left|\frac{1}{N} \sum_{j=1}^{N} \exp \left(i \psi_{j}\right)\right|
$$

where

$$
\psi_{j}(t)=2 \pi m+2 \pi \frac{t-t_{j, m}}{t_{j, m+1}-t_{j, m}},
$$

where $t_{j, m}$ denotes when a spike $m(m=0,1,2, \ldots)$ of a neuron $j$ occurs $\left(t_{j, m}<t<t_{j, m+1}\right)$. The beginning of each spike is considered when $V_{j}>0$. If the spikes times are uncorrelated, their contribution to the result of the summation is small [38]. However, in a globally synchronised state the order parameter magnitude asymptotes the unity.

Figure 3(a) shows the spiking patterns for $p=0.1$, and without external perturbation, that is, the neuronal network presents asynchronous dynamics, where the points correspond to spiking neurons, and the absent points correspond to the resting neurons. For $p=1.0$, we have globally coupled neurons, and we can see that the network exhibits synchronised spiking, shown in Fig. 3(b). The time evolution of the order parameter is plotted in Fig. 3(c). When $p=0.1$ (black line), the network does not display synchronised behaviour, and as a result the order parameter is typically small with $R$ fluctuating around 0.1. However, synchronised behaviour is observed for $p=1.0$ with order parameter values near unity (red line).

We add an external perturbation $\left(\Gamma_{i}\right)$ to analyse its effects on the synchronous behaviour. This way we compute the time averaged magnitude of 
the order parameter, given by

$$
\bar{R}=\frac{1}{t_{\text {fin }}-t_{\text {ini }}} \sum_{t=t_{\text {ini }}}^{t_{\text {fin }}} R(t),
$$

where the values of $\bar{R}$ have been computed by averaging over a temporal length of $10 \mathrm{~s}$ after discarding a transient of $490 \mathrm{~s}\left(t_{\text {ini }}=490 \mathrm{~s}\right.$ and $t_{\text {fin }}=$ 500s). In Fig. 4 we can see the time averaged order parameter as a function of the probability for different input amplitudes $(\gamma)$. When the neuronal network has no input, it is possible to observe synchronised behaviour if the probability of connection is large enough (black circles). However, external inputs are able to desynchronise the spiking neurons, as shown in Fig. 4 for $\gamma=5$ (red triangles) and $\gamma=10$ (blue squares), where the values of $\bar{R}$ are less than 0.9 .

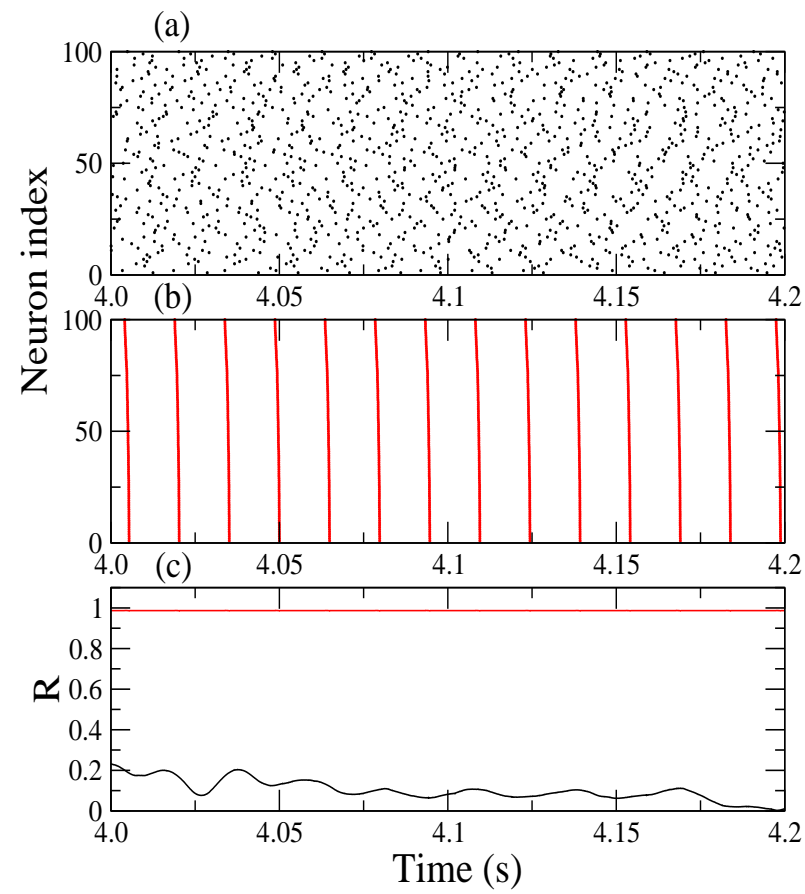

Figure 3: (Colour online) Space-time plots of the membrane potentials for $N=100$, (a) $p=0.1$, and (b) $p=1.0$. In (c) the order parameter is calculated for $p=0.1$ (black line), and $p=1.0$ (red line). 


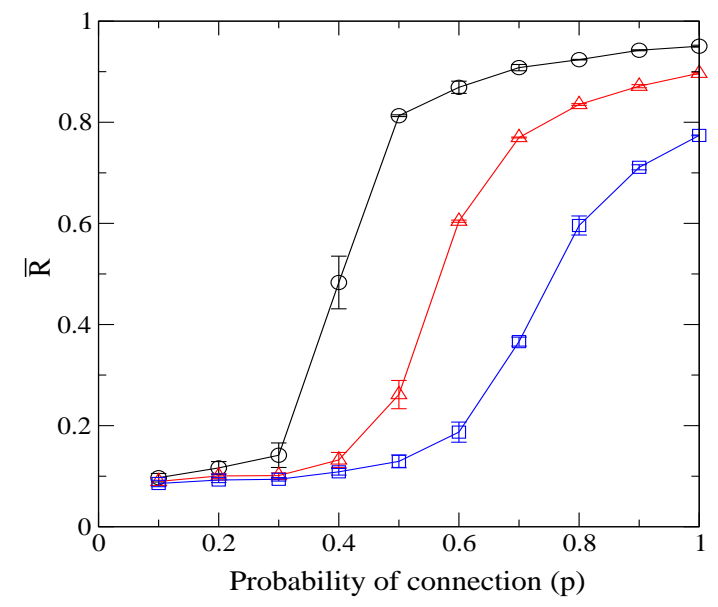

Figure 4: (Colour online) Average order parameter as a function of the probability, for $N=100$, for external perturbation with $\gamma=0$ (black circles), $\gamma=5$ (red triangles), and $\gamma=10$ (blue squares). The bars represent the standard deviation.

\section{Spike timing-dependent plasticity}

One of the key principles of behavioural neuroscience is that experience can modify the brain structure, that is known as neuroplasticity [39]. Although the idea that experience may modify the brain structure can probably be traced back to the 1890s [40, 41], it was Hebb who made this a central feature in his neuropsychological theory [42].

With this in mind, we consider spike timing-dependent plasticity according to the Hebbian rule. In this plasticity the coupling strength $\varepsilon_{i j}$ is adjusted based on the relative timing between the spikes of pre-synaptic and post-synaptic neurons [25].

$$
\Delta \varepsilon_{i j}=\left\{\begin{array}{l}
A_{1} \exp \left(-\Delta t_{i j} / \tau_{1}\right), \Delta t_{i j} \geq 0 \\
-A_{2} \exp \left(\Delta t_{i j} / \tau_{2}\right), \Delta t_{i j}<0
\end{array},\right.
$$

where $\Delta t_{i j}=t_{i}-t_{j}=t_{\text {pos }}-t_{\text {pre. }}$. Figure 5 exhibits the result that is obtained from Eq. (16) for $A_{1}=1.0, A_{2}=0.5, \tau_{1}=1.8 \mathrm{~ms}$, and $\tau_{2}=6.0 \mathrm{~ms}$.

The initial synaptic weights $\varepsilon_{i j}$ are normally distributed with mean and variance equal to 0.1 and 0.02 , respectively. Then, they are updated according to Eq. (16), where $\varepsilon_{i j} \rightarrow \varepsilon_{i j}+10^{-3} \Delta \varepsilon_{i j}$. In the absence of an external perturbation, we can verify by means of Fig. 6(a) that the averaged synaptic weights can be depressed $(p=0.3)$ or potentiated $(p=0.5, p=0.7$, and 


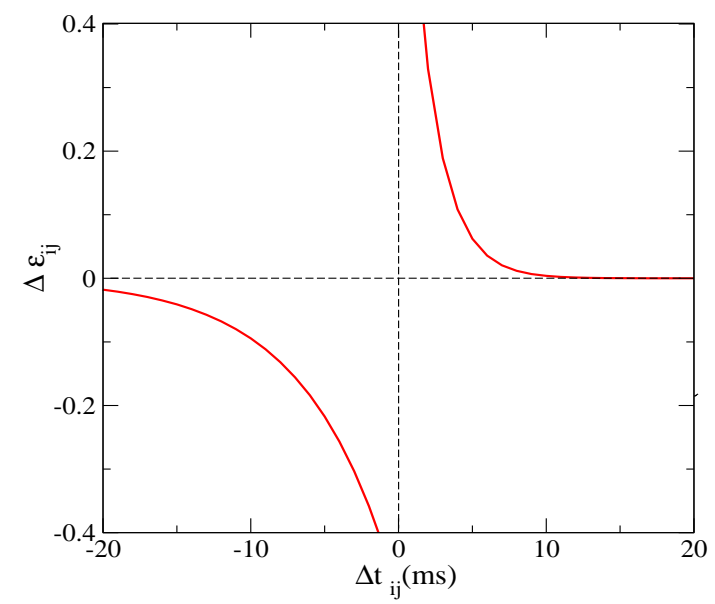

Figure 5: Plasticity function (16) as a function of the difference of spike timing of postand pre-synaptic neuron.

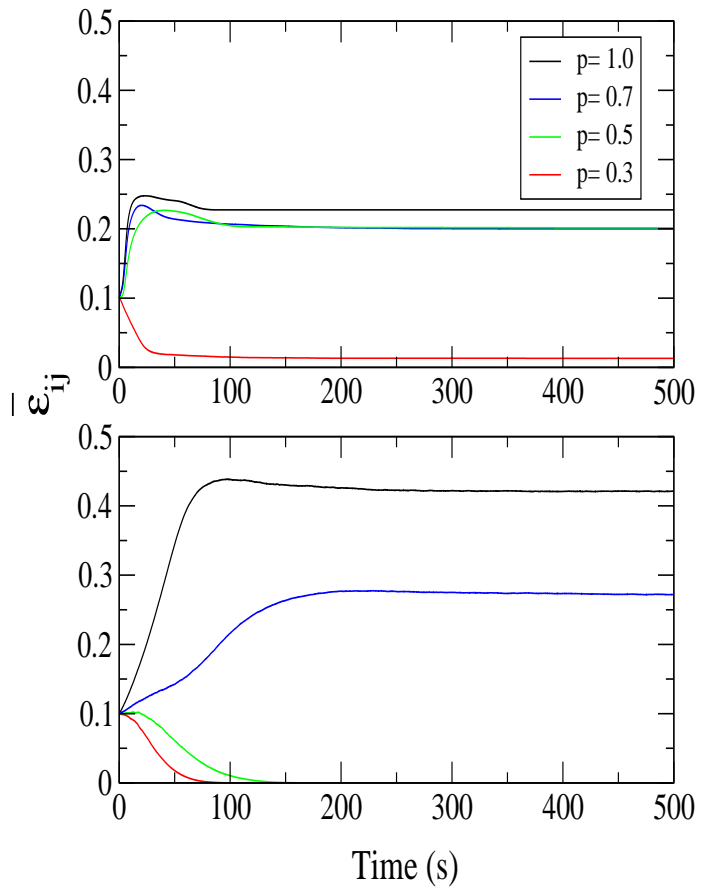

Figure 6: (Colour online) Time evolution of the averaged coupling strength for $p=0.3$ (red line), $p=0.5$ (green line), $p=0.7$ (blue line), and $p=1.0$ (black line), where it is considered (a) $\gamma=0$, and (b) $\gamma=5$. 
$p=1.0)$ depending on the probability of connections. If an input is applied on the neuronal network (Fig. 6b), the input can have a constructive effect on the synaptic weights $(p=0.7$ and $p=1.0)$ or destructive effect $(p=0.3$ and $p=0.5$ ), depending on the probability of connections.
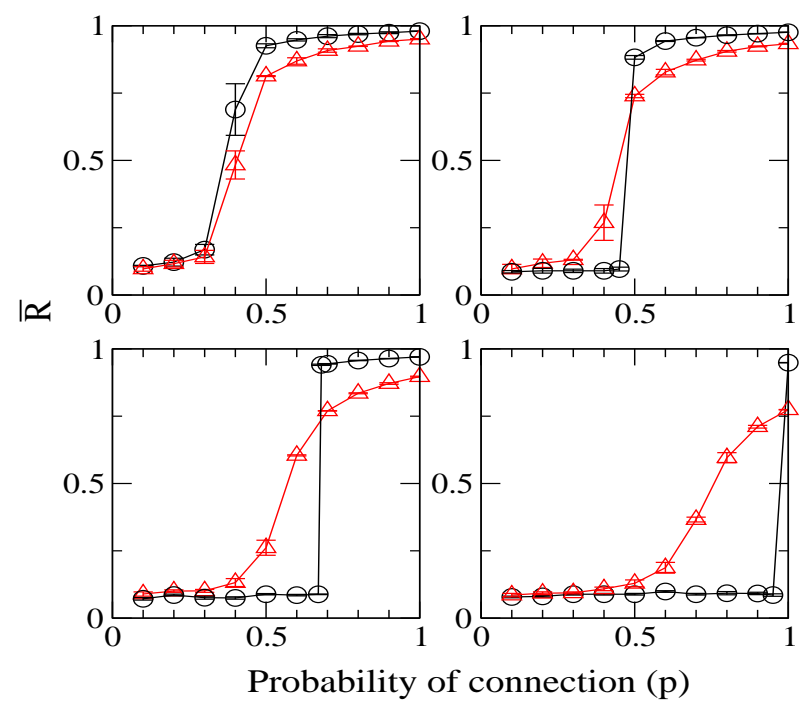

Figure 7: (Colour online) Average order parameter versus probability of connection for the cases with (black circles) and without (red triangles) STDP, where we consider (a) $\gamma=0$, (b) $\gamma=3$, (c) $\gamma=5$, and (d) $\gamma=10$. The bars represent the standard deviation.

The time averaged order parameter in terms of the probability of connection is showed in Fig. 7, considering with (black circles) and without (red triangles) STDP. In the case for without external perturbation (Fig. Tha) we can see that the values of $\bar{R}$ without STDP are less than with STDP, namely STDP is producing a positive effect on the synchronisation. Increasing the amplitude of the external perturbation, without spike timing-dependent plasticity, the desynchronisation is induced in the neuronal network (Fig. 44). However, considering STDP in the perturbed network is possible to observe alterations to the dynamic behaviour in relation to synchronised states. Figure 7 (b) shows that the STDP enhances the synchronisation for $p$ approximately greater than 0.5 due to a constructive effect on the dynamics of the synaptic weights. On the other hand, for $p$ less than 0.5 the STDP decreases the values of the time averaged order parameter, as a result of depressed synaptic weights. Increasing the input intensity $(\gamma=5)$, it is possible to 
verify synchronisation when the network has neuroplasticity (Fig. 76). We can also see an abrupt transition from a desynchronised to a synchronised regime. When the input intensity increases, it is necessary to increase the probability of connection for the STDP counteracts the suppression of synchronisation. Figure $7(d)$ exhibits a situation such that the synchronisation is only obtained when the neuronal network presents a global coupling $(p=1)$. With a strong input, the STDP does not lead the network to a potentiation of synaptic weights, and this way the synchronisation is suppressed by an external input.
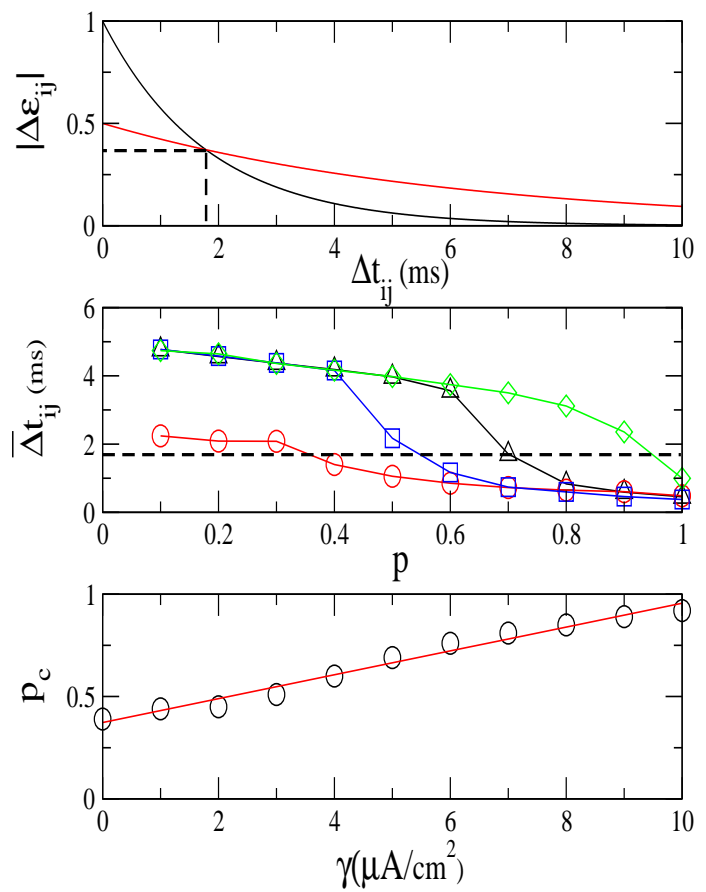

Figure 8: (Colour online) (a) Absolute value of the plasticity function (16) as a function of the difference of spike timing of post- and pre-synaptic neuron, where the curves are the potentiation (red line) and the depression (black line). (b) Average time difference versus probability of connections for $\gamma=0$ (red circles), $\gamma=3$ (blue squares), $\gamma=5$ (black triangles), and $\gamma=10$ (green diamonds). (c) Critical probability $p_{c}$ as a function of the input level $\gamma$. The linear fit is given by the equation $p_{c}=0.06 \gamma+0.37$.

The spike synchronisation depends on the probability of connections $p$ in a way showing an abrupt transition. There is a critical point for $p=p_{c}$ that can be found by means of the intersection between the curves of potentiation 
and depression. Figure 8 (a) exhibits the point of intersection with value of $\Delta t_{i j}$ approximately equal to 1.8. For $\Delta t_{i j}>1.8$ the depression (red line) of the synaptic strength is larger than the potentiation (black line), while that for $\Delta t_{i j}<1.8$ the potentiation is larger than the depression. With this value of $\Delta t_{i j}$ we can obtain $p_{c}$ plotting $\Delta \bar{t}_{i j}$ as a function of $p$, as shown in Fig. 8 (b). It can be seen that the value of $\Delta \bar{t}_{i j}$ decays, and when cross the value $\Delta \bar{t}_{i j} \approx 1.8$ we have the critical value of the probability of connections. Moreover, when $p$ increases not only $\Delta \bar{t}_{i j}$ decreases, but also the variance of the inter-spike intervals decreases. Then, we compute the critical probability as a function of the input level (Fig. 86), where we verify a linear increase given by the equation $p_{c}=0.06 \gamma+0.37$.

Figure 7)(c) shows a discontinuous transition between the synchronised and the desynchronised regime. Our aim is to understand how this discontinuous transition appears when the probability of connections is varied. For this reason, we build a network according to a schematic representation that is showed in Fig. 9. The scheme represents a network of neurons with high (cyan ball) and low (yellow ball) spiking frequency. The red arrows represent the connections between neurons from high to low frequency, while the black arrows represent the connections from low to high frequency.

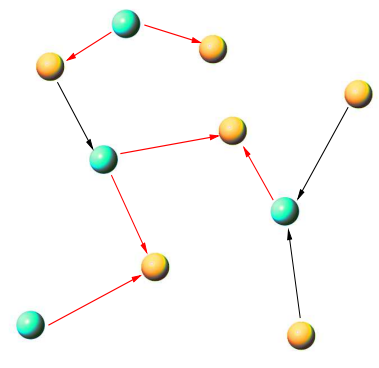

Figure 9: (Colour online) Schematic representation of a network of neurons with high (cyan ball) and low (yellow ball) spiking frequency. The red arrows represent the connections between neurons from high to low frequency, while the black arrows represent the connections from low to high frequency.

Based on the schematic representation that is illustrated in Fig. 9, we consider a neuronal network with $N=100, p=0.47$, and $\gamma=3$. We separate the neuronal network into 50 neurons with high frequency (values of $I_{i}$ within the range $[9.0,9.1]$ ) and 50 neurons with low frequency (values of $I_{i}$ within 
the range $[9.9,10])$. Figure 10 exhibits the time averaged coupling strength as a function of the percentage of connections from neurons with high frequency to neurons with low frequency. We can see that the time average coupling strength depends on the connections. Considering the case for a small percentage of connections from HFN to LFN, the average coupling is small, indicating absence of synchronisation, a situation that changes with increasing the percentage of connections to a synchronised state. This means that, when the coupling strengths increase, a desynchronised state can suddenly become synchronised. Consequently, the abrupt transition from desynchronised to synchronised state, that is observed in Fig. 7, is due to directed synapses among spiking neurons with high and low frequency.

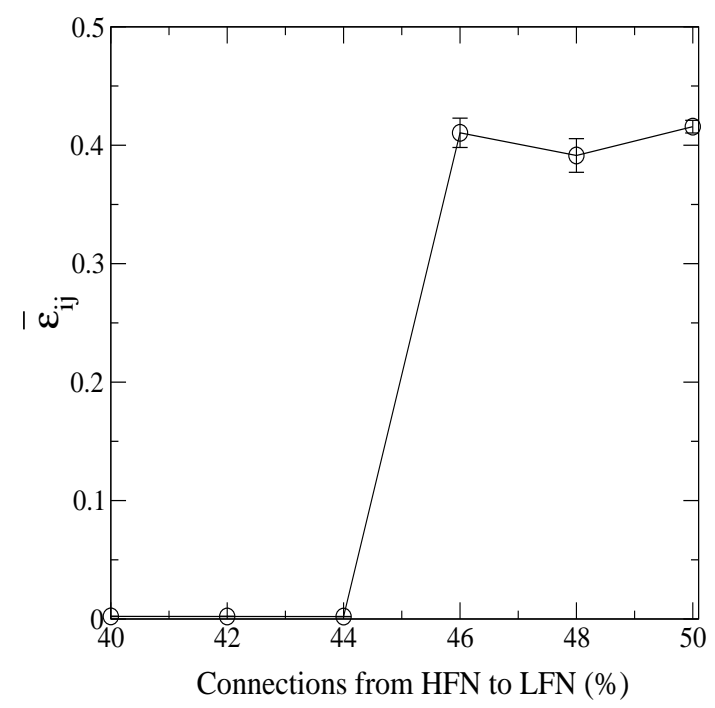

Figure 10: Time averaged coupling strength versus percentage of connections from neurons with high frequency (HFN) to neurons with low frequency (LFN). We consider $p=0.47$ and $\gamma=3$.

\section{Conclusion}

We have been studying a neuronal network model with spiking neurons. We have chosen, as local dynamics, the Hodgkin-Huxley model due to the fact that it has essential features of spiking dynamics. The Hodgkin-Huxley model is a coupled set of nonlinear differential equations that describes the 
ionic basis of the action potential. These equations are able to reproduce biophysical properties of the action potential.

We have used a random coupling architecture where the connections are randomly distributed according to a probability. When the probability is equal to unity we have a globally coupled network. The connections were considered unidirectional representing excitatory chemical synapses.

We have studied the effects of spike timing-dependent plasticity on the synchronisation in a Hodgkin-Huxley neuronal network. Studies about spike synchronisation are important to understand not only progressively degenerative neurological disorders, but also processing of the sensory information. Popovych and collaborators [20] showed that STDP combined with an external perturbation can improve the spike synchronisation in a globally neuronal network. The novelty in this paper is that we have considered a random neuronal network and we have verified that the spike synchronisation depend on the probability of connections. Considering a strong external perturbation the spike synchronisation is suppressed. However, when there is STDP, depending on the probability of connections, the synchronisation in the perturbed network can be improved due to a constructive effect on the synaptic weights.

We have also shown that the direction of synapses has an important role on the effects of spike timing-dependent plasticity on the synchronisation in a random Hodgkin-Huxley neuronal network.

\section{Acknowledgements}

This study was possible by partial financial support from the following Brazilian government agencies: CNPq, CAPES and FAPESP. Financial support by the Spanish Ministry of Economy and Competitivity under project number FIS2013-40653-P is also acknowledged.

\section{References}

[1] Chialvo DR. Critical brain networks. Phys A 2004;340:756-765.

[2] Gerstner W, Kistler W. Spiking neuron models: Single neurons, populations, plasticity. Cambridge University Press: Cambridge; 2002.

[3] Bear MF, Connors BW, Paradiso MA. Neuroscience: Exploring the brain. Lippincott Williams and Wilkins: England; 2008. 
[4] Byrne JH. Fundamental neuroscience. Academic Press: New York; 2008.

[5] Dayan P, Abbott LF. Theoretical neuroscience: Computational and mathematical modelling of neural systems. Mit Press: Massachusetts; 2001.

[6] Purves D, Augustine DJ, Fitzpatrick D, Hall WC, LaMantia A-S, McNamara JO, Williams SM. Neuroscience. Sinauer Associates Inc Publishers: Massachusetts: 2004.

[7] Dagostin AA, Mello CV, Leão RM. Increased bursting glutamatergic neurotransmission in an auditory forebrain area of the zebra finch (Taenopygia guttata) induced by auditory stimulation. J Comp Physiol A 2012:198;705-716.

[8] Hebb DO. The organization of behavior. Wiley: New York; 1949.

[9] Citri A, Malenka RC. Synaptic plasticity: multiple forms, functions, and mechanisms. Neuropsychopharmacol 2008:33;18-41.

[10] Kelso SR, Ganong AH, Brown TH. Hebbian synapses in hippocampus. Proc Natl Acad Sci USA 1986:83;5326-5330.

[11] Caporale N, Dan Y. Spike timing-dependent plasticity: a Hebbian learning rule. Annu Rev Neurosci 2008:31;25-46.

[12] Zhigulin VP, Rabinovich MI, Huerta R, Abarbanel HD. Robustness and enhancement of neural synchronization by activity-dependent coupling. Phys Rev E 2003:67;021901.

[13] Abuhassan K, Coyle D, Maguire L. Compensating for thalamocortical synaptic loss in Alzheimers disease. Front Comput Neurosci 2014:8;65.

[14] Modolo J, Bhattacharya B, Edwards R, Campagnaud J, Legros A, Beuter A. Using a virtual cortical module implementing a neural field model to modulate brain rhythms in Parkinsons disease. Front Neurosci 2010:4;45.

[15] Hammond C, Bergman H, Brown P. Pathological synchronization in Parkinsons disease: networks, models and treatments. Trends Neurosci 2007:30;357-364. 
[16] Nini A, Feingold A, Slovin H, Bergman H. Neurons in the globus pallidus do not show correlated activity in the normal monkey, but phase-locked oscillations appear in the MPTP model of parkinsonism. J Neurophysiol 1995:74;1800-1805.

[17] Uhlhass P, Singer W. Neural synchrony in brain disorders: relevance for cognitive dysfunctions and pathophysiology. Neuron 2006:52;155-168.

[18] Lameu EL, Batista CAS, Batista AM, Iarosz KC, Viana RL, Lopes SR, Kurths J. Suppression of bursting synchronization in clustered scale-free (rich-club) neuronal networks. Chaos 2012:22;043149.

[19] Popovych OV, Tass PA. Desyncrhonizing electrical and sensory coordinated reset neuromodulation. Front Hum Neurosci 2012:6;58.

[20] Popovych OV, Yanchuk S, Tass PA. Self-organized noise resistance of oscillatory neural networks with spike timing-dependent plasticity. Sci Rep 2013:3;2926.

[21] Nordenfelt A, Used J, Sanjuán MAF. Bursting frequency versus phase synchronization in time-delayed neuron networks. Phys Rev E 2013:87;052903.

[22] Tass PA, Silchenko AN, Hauptmann C, Barnikol UB, Speckmann EJ. Long-lasting desynchronization in rat hippocampal slice induced by coordinated reset stimulation. Phys Rev E 2009:80;011902.

[23] Feldman DE. The spike-timing dependence of plasticity. Neuron 2012:75;556-571.

[24] Markram H, Gerstner W, Sjöström PJ. Spike-timing-dependent plasticity: A comprehensive overview. Front Synaptic Neurosci 2012:4;8.

[25] Bi GQ, Poo MM. Synaptic modifications in cultured hippocampal neurons: dependence on spike timing, synaptic strength, and postsynaptic dell type. J Neurosci 1998:18;10464-10472.

[26] Gerstner W, Sprekeler H, Deco G. Theory and simulation in neuroscience. Sci 2012:338;60-65.

[27] Gray EG. Electron microscopy of synaptic contacts on dendrite spines of the cerebral cortex. Nature 1959:183;1592-1593. 
[28] Hodgkin AL, Huxley AF. A quantitative description of membrane current and its application to conduction and excitation in nerve. J Physiol 1952:117;500-544.

[29] Izhikevich EM. Which model to use for cortical spiking neurons? IEEE T Neur Net 2004:15;1063-1070.

[30] Izhikevich EM. Dynamical systems in neuroscience: The geometry of excitability and bursting. MIT Press: London; 2006.

[31] Erdös P, Rényi A. On random graphs I. Publ Math 1959:6;290-297.

[32] Nordenfelt A, Wagemakers A, Sanjuán MAF. Frequency dispersion in the time-delayed Kuramoto model. Phys Rev E 2014:89;032905.

[33] Nordenfelt A, Wagemakers A, Sanjuán MAF. Cyclic motifs as the governing topological factor in time-delayed oscillator networks. Phys Rev E 2014:90;052920.

[34] Destexhe A, Mainen ZF, Sejnowki TJ. An efficient method for computing synaptic conductances based on a kinetic model of receptor binding. Neural Comput 1994:6;14-18.

[35] Golomb D, Rinzel J. Dynamics of globally coupled inhibitory neurons with heterogeneity. Phys Rev E 1993:48;4810.

[36] Kuramoto Y. International Symposium on mathematical problems in theoretical physics. Springer-Verlag: New York; 1975.

[37] Kuramoto Y. Chemical oscillations, waves, and turbulence. Springer: Berlin; 1984.

[38] Acebrón JA, Bonilla LL, Vicente CJP, Ritort F, Spigler R. The Kuramoto model: A simple paradigm for synchronization phenomena. Rev Mod Phys 2005:77;137-185.

[39] Ramon Y Cajal S. Degeneration and Regeneration of the Nervous System. Oxford University Press: London; 1928.

[40] Bliss TVP, Gardner-Medwin AR. Long-lasting potentiation of synaptic transmission in the Dentate Area of the unanaestherised rabbit following stimulation of the perforant path. J Physiol 1973:232;357-374. 
[41] Bliss TVP, Collingridge GL. A synaptic model of memory: long-term potentiation in the hippocampus. Nature 1993:361;31-39.

[42] Hebb DO. Brain mechanisms and learning. Oxford University Press: London; 1961. 